\title{
Jovian Problem: Performance of Some High-Order Numerical Integrators
}

\author{
Shafiq Ur Rehman ${ }^{1,2}$ \\ ${ }^{1}$ Department of Mathematics, The University of Auckland, Auckland, New Zealand, \\ ${ }^{2}$ Department of Mathematics, University of Engineering and Technology, Lahore, Pakistan \\ Email: s.rehman@math.auckland.ac.nz,srehman@uet.edu.pk
}

Received July 3, 2013; revised August 1, 2013; accepted August 9, 2013

Copyright (C) 2013 Shafiq Ur Rehman. This is an open access article distributed under the Creative Commons Attribution License, which permits unrestricted use, distribution, and reproduction in any medium, provided the original work is properly cited.

\begin{abstract}
$\mathrm{N}$-body simulations of the Sun, the planets, and small celestial bodies are frequently used to model the evolution of the Solar System. Large numbers of numerical integrators for performing such simulations have been developed and used; see, for example, $[1,2]$. The primary objective of this paper is to analyse and compare the efficiency and the error growth for different numerical integrators. Throughout the paper, the error growth is examined in terms of the global errors in the positions and velocities, and the relative errors in the energy and angular momentum of the system. We performed numerical experiments for the different integrators applied to the Jovian problem over a long interval of duration, as long as one million years, with the local error tolerance ranging from $10^{-16}$ to $10^{-8}$.
\end{abstract}

Keywords: $N$-Body Simulations; Jovian Problem; Numerical Integrators; CPU-Time

\section{Introduction}

Computational astronomers make extensive use of accurate $N$-body simulations when studying the dynamics of the planets, asteroids and other small celestial bodies in the Solar System. These simulations are performed by first deriving a set of differential equations for the acceleration of the $N$ bodies in the simulation, and specifying the initial positions and velocities of the bodies at time $t$ $=t_{0}$. Generally, the initial value problems (IVPs) that occur for $N$-body simulations are a mixture of first- and second-order differential equations, but the sort of problems we are considering are of the form,

$$
y^{\prime \prime}(t)=f(t, y(t)), y\left(t_{0}\right)=y_{0}, y^{\prime}\left(t_{0}\right)=y_{0}^{\prime},
$$

where $y_{0} \in \mathbb{R}^{k}$ and $y_{0}^{\prime} \in \mathbb{R}^{k}$ denote the initial positions and velocities, the operator denotes differentiation with respect to time $t$, and $f: \mathbb{R} \times \mathbb{R}^{k} \rightarrow \mathbb{R}^{k}$ is a sufficiently smooth function. Here, $k$ is the dimension of the IVP, which in some cases may change over time, as bodies are added or removed in the simulations. In some cases, these equations can be solved analytically, but mostly the differential equations are too complicated to find analytical solutions, necessitating the use of approximation techniques to find the numerical approximate solution. A wide range of integrators, for example, Runge-Kutta [3,4], Linear multistep [5], Runge-Kutta-
Nyström [6], and Störmer [7] are used to find a numerical solution to the differential equations at $t=t_{0}+i h$, with $i=1,2, \cdots$ and time-step $h$, which can depend on $i$.

\section{Jovian Problem}

The Jovian problem (see, for example, [1]) models the orbital motion of the Sun and the four Gas giants, Jupiter, Saturn, Uranus and Neptune, interacting through Newtonian gravitational forces. The Jovian problem is often used in numerical experiments, because the Gas giants collectively drive much of the dynamics of the Solar System. Let $r_{i}=\left[x_{i}, y_{i}, z_{i}\right]^{T}, i=1, \cdots, 5$, be the position vector of the $i^{\text {th }}$ body of the Jovian problem, where the bodies are ordered from Sun to Neptune and the coordinate system is the three-dimensional Cartesian system with the origin at the barycentre (centre of mass) of the bodies. Then the equations of motion for the $i^{\text {th }}$ body can be written as

$$
r_{i}^{\prime \prime}(t)=\sum_{j=1, j \neq i}^{5} \frac{\mu_{j}\left(r_{j}(t)-r_{i}(t)\right)}{\left\|r_{j}(t)-r_{i}(t)\right\|_{2}^{3}}, i=1, \cdots, 5,
$$

where $\|\cdot\|_{2}$ denotes the $L_{2}$-norm and $\mu_{j}$ is the gravitational constant $G$ times the mass $m_{j}$ of the $j^{\text {th }}$ body, i.e, $\mu_{j}=G m_{j}$. For each body we have a second-order differential equation for the $x$-, $y$-, and $z$-component, 
giving us fifteen second-order differential equations in total. We express units of distance in astronomical units, the independent variable $t$ in Earth days and the mass $m_{j}$ in Solar mass.

We use the symmetry of interactions to reduce the number of calculations in the subroutine for evaluating the acceleration for the Jovian problem. Consider the individual terms in the summation and observe

$$
\frac{\left(r_{j}(t)-r_{i}(t)\right)}{\left\|r_{j}(t)-r_{i}(t)\right\|_{2}^{3}}=\frac{-\left(r_{i}(t)-r_{j}(t)\right)}{\left\|r_{j}(t)-r_{i}(t)\right\|_{2}^{3}} .
$$

Hence, once this term for $r_{j}$ is calculated, we can update the acceleration for the second body by symmetry. Using this symmetry, we found that the subroutine for the evaluation of the force term for the Jovian problem reduces to approximately half of the CPU-time.

Unlike the Kepler problem, an analytical solution for the Jovian problem is unavailable. Therefore, numerical experiments using the Jovian problem require a reference solution in order to obtain an estimate of the error in the position and velocity. The reference solution has to be more accurate than the numerical solution. Since we plan to test the numerical integrators near the limit of doubleprecision arithmetic $\left(2.2 \times 10^{-16}\right)$, it is essential to use quadruple-precision arithmetic for the reference solution. Therefore, for long-term simulations, obtaining a reference solution can require considerable CPU-time.

Different types of errors are discussed throughout this paper. The global error is of major importance in the measurement of the quality of the numerical solution. We measure this global error in position and velocity, and also measure the relative error in energy and angular momentum. For the total error in the system the main source of error is the integration error, which consists of a truncation and round-off error. While performing accurate simulations, the round-off error contributes significantly to the global error because computers store numbers to only a certain precision. So, there will always be a loss of accuracy when performing long-term simulations. For fixed-step-size schemes, Brouwer [8] showed that, if the step-size is smaller than a prescribed value, the round-off error for conserved quantities, such as total energy and angular momentum, grows as $t^{\frac{1}{2}}$ and for other dynamical variables, such as coordinates of particles, as $t^{\frac{3}{2}}$. This error growth is known as Brouwer's law in the literature; see, for example, [9,10]. In contrast, when the round-off error is systematic, the power laws become $t$ and $t^{2}$, respectively. In addition to these aspects, we investigate other effects of the round-off error here.

First we define the types of errors used in this paper.
Let $y_{n}$ and $y_{t}$ be the vectors of the solution calculated numerically and the reference solution, respectively, and $y_{n}^{\prime}$ and $y_{t}^{\prime}$ are the vectors of the derivative to the numerical and reference solutions, respectively. Then the norm of the global errors in the position and the velocity are given by

$$
E_{r}(t)=\left\|y_{n}-y_{t}\right\|_{2}, E_{v}(t)=\left\|y_{n}^{\prime}-y_{t}^{\prime}\right\|_{2},
$$

where, \|\|$_{2}$ is the unweighted $L_{2}$-norm.

Physical systems often have conserved quantities, for example, the total energy $H$ or the total angular momentum $L$ as for Kepler's two-body problem and the Jovian problem. Usually, these quantities will not be conserved exactly by the numerical solution and this derivation provides assessment about the accuracy of the solution. The total energy is defined as

$$
H=\frac{1}{2} \sum_{i=1}^{N} m_{i} v_{i}^{2}-\sum_{j=1}^{N-1} \sum_{i=j+1}^{N} G \frac{m_{i} m_{j}}{d_{i j}},
$$

where $G$ is the gravitational constant, $m_{i}$ the mass of the $i^{\text {th }}$ body, $v_{i}$ its velocity, and $d_{i j}=\left\|r_{i}-r_{j}\right\|_{2}$ the distance between the $i^{\text {th }}$ and $j^{\text {th }}$ bodies. The relative error in the energy can be calculated as

$$
H_{\text {rel }}=\left|\frac{H_{0}-H}{H_{0}}\right|,
$$

where $H_{0}$ is the total energy at the initial time $t=0$. However, we use

$$
H_{r e l}=\left|\frac{G H_{0}-G H}{G H_{0}}\right|,
$$

to calculate $H_{\text {rel }}$, because the value of $\mu=G m$ is known more accurately than $m$.

The total angular momentum $L$ and the relative error of the angular momentum $L_{r e l}$ are defined as

$$
L=\sum_{i=1}^{N} m_{i} r_{i} \times v_{i}, L_{r e l}=\frac{\left\|L_{0}-L\right\|_{2}}{\left\|L_{0}\right\|_{2}},
$$

where $L_{0}$ is the angular momentum at the initial time $t=0$. Note that a reference solution is not required to calculate $H_{r e l}$ and $L_{r e l}$, unlike for the errors in the position and velocity. Hence, less computing resources are needed to measure the performance of the integrators here. However, $H_{r e l}$ and $L_{r e l}$, being scalar quantities, impose only one constraint; if we look at the error in position or velocity then every component of $E_{r}$ or $E_{v}$ has to be small.

The phase error is the difference between the phase angle of the numerical solution and the reference solution. The phase error is defined by

$$
\cos (\theta)=\frac{y_{n} \cdot y_{t}}{\left\|y_{n}\right\|_{2}\left\|y_{t}\right\|_{2}} .
$$


where $\theta$ is the angle between the numerical solution and the reference solution.

\section{Numerical Methods and Integrators}

Explicit Runge-Kutta-Nyström methods (ERKN) were introduced by E. J. Nyström in 1925 [6]. The efficiency of an ERKN method depends upon the approach for controlling the error in the numerical approximations. One way of controlling the error is to use an adaptive step-size technique. In order to control the local error of a single step, a pair of formulae of different orders is used in such a way that the function evaluations of the two methods are identical. If the numerical solution $y_{n}$ is obtained by using the lower-order formula, then the pair is said to be implemented in lower-order mode. However, it is recommended for efficiency reasons that the solution $y_{n}$ be obtained using the higher-order formula for the next step [11], and the pair operated in this fashion is said to be implemented in higher-order mode or local extrapolation. In this paper we are using two variable-step-size ERKN integrators: Integrator ERKN689 is a nine stage, 6-8 FSAL pair [12] and integrator ERKN101217 is a seventeen stage, 10-12 non-FSAL pair [12].

\subsection{Round-Off Error Control for ERKN Integrators}

In this paper, we perform experiments with tolerance close to the machine precision $\left(2.2 \times 10^{-16}\right)$. Therefore, we investigate the possibility of reducing the growth of round-off error in the explicit Runge-Kutta-Nyström integrators using the technique known as compensated summation [13]. The idea of compensated summation is based on estimating the dominant contribution term of the round-off error. To explain the round-off error control technique, we consider the following solution formula

$$
y_{n}=y_{n-1}+h y_{n-1}^{\prime}+h^{2} \sum_{j=1}^{s} b_{j} K_{j}
$$

Equation (1.3) contains three types of errors; the integration error already in $y_{n}$ from the previous timestep, the round-off error in the formation of $h y_{n-1}^{\prime}$ and $h^{2} \sum_{j=1}^{s} b_{j} K_{j}$, and the round-off error caused by adding terms on the right-hand side of (1.3). If the integration time-step is small then $h y_{n-1}^{\prime}+h^{2} \sum_{j=1}^{s} b_{j} K_{j}$ is small compared to $y_{n-1}$. Hence, the round-off error will be dominated by adding $h y_{n-1}^{\prime}+h^{2} \sum_{j=1}^{s} b_{j} K_{j}$ to $y_{n-1}$. In each time-step we estimate the round-off error caused by adding $h y_{n-1}^{\prime}+h^{2} \sum_{j=1}^{s} b_{j} K_{j}$ to $y_{n-1}$ and then update the solution as follows; First, calculate

$$
\tau=h y_{n-1}^{\prime}+h^{2} \sum_{j=1}^{s} b_{j} K_{j}-\delta,
$$

where $\delta$ is the estimated round-off error on the previous time-step (at the start of the integration $\delta=0$ ). Since $h y_{n-1}^{\prime}+h^{2} \sum_{j=1}^{s} b_{j} K_{j}$ and $\delta$ are small compared to $y_{n-1}$, the error caused in the formation of $\tau$ is negligible. The solution is then updated to

$$
Y_{n}=y_{n-1}+\tau,
$$

and the estimated round-off error for the time-step is calculated as

$$
\delta=Y_{n}-y_{n}-\tau
$$

The solution is then updated as $y_{n}=Y_{n}$. The velocity formula also uses the same concept to control the roundoff error.

We used the round-off error control technique to investigate the maximum error in position $\left(E_{r}\right)$ and velocity $\left(E_{v}\right)$ for the Jovian problem described in Section 2 . The integration was performed over $10^{6}$ years using $T O L=10^{-2 i}$, for $i=4,5, \cdots, 8$. Table 1 shows the maximum values of $E_{r}$ and $E_{v}$ for the explicit RungeKutta-Nyström integrators ERKN689 and ERKN101217. The column labelled With contains $E_{r}$ and $E_{v}$ calculated when the integration is performed with round-off error control, whereas the column labeled Without contains the percentage variation corresponding to the values in column With when calculated $E_{r}$ and $E_{v}$ by performing integration with-out round-off error control.

For $E R K N 689$, the maximum values for $E_{r}$ and $E_{v}$ with round-off error control are always less than $E_{r}$ and $E_{v}$ without round-off error control. The only exception is for $T O L=10^{-10}$, where the values of $E_{r}$ and $E_{v}$ in the

Table 1. The maximum values of $E_{r}$ and $E_{v}$ for $E R K N 689$ and $E R K N 101217$ obtained with and with-out round-off error control applied to the Jovian problem over one million years for the local error tolerances $10^{-8}, 10^{-10}, 10^{-128}, 10^{-14}, 10^{-16}$.

\begin{tabular}{ccccccccc}
\hline \multicolumn{9}{c}{ ERKN689 } \\
\hline \multicolumn{3}{c}{$E_{r}$} & \multicolumn{3}{c}{$E_{v}$} & \multicolumn{3}{c}{ ERKN101217 } \\
\hline TOL & With & Without & With & Without & With & Without & With & Without \\
\hline $10^{-8}$ & $4.37 \times 10^{-2}$ & $+0.02 \%$ & $6.31 \times 10^{-5}$ & $+0.02 \%$ & $4.70 \times 10^{-1}$ & $+0.21 \%$ & $6.78 \times 10^{-4}$ & $+0.29 \%$ \\
$10^{-10}$ & $1.11 \times 10^{-4}$ & $-0.91 \%$ & $1.60 \times 10^{-7}$ & $-0.63 \%$ & $1.63 \times 10^{-3}$ & $-0.62 \%$ & $2.35 \times 10^{-6}$ & $-0.86 \%$ \\
$10^{-12}$ & $1.70 \times 10^{-6}$ & $+71.8 \%$ & $1.94 \times 10^{-9}$ & $+68.9 \%$ & $4.82 \times 10^{-5}$ & $-0.21 \%$ & $6.63 \times 10^{-8}$ & $-0.15 \%$ \\
$10^{-14}$ & $9.74 \times 10^{-7}$ & $+86.0 \%$ & $9.35 \times 10^{-10}$ & $+84.4 \%$ & $2.42 \times 10^{-5}$ & $-6.61 \%$ & $3.33 \times 10^{-8}$ & $-7.77 \%$ \\
$10^{-16}$ & $2.28 \times 10^{-6}$ & $+71.0 \%$ & $1.58 \times 10^{-9}$ & $+78.5 \%$ & $8.71 \times 10^{-6}$ & $-7.40 \%$ & $1.19 \times 10^{-8}$ & $-6.25 \%$ \\
\hline
\end{tabular}


column Without are close to zero and insignificant compared with values for smaller tolerances. The maximum difference was observed for $T O L=10^{-14}$. Here, the maximum values for $E_{r}$ and $E_{v}$ obtained with roundoff error control were approximately $86 \%$ and $84 \%$ less than those obtained without round-off error control. For $E R K N 101217$, except for $T O L=10^{-8}$, we found that the round-off error control technique is not very effective, because the errors in the position and velocity obtained with round-off error control are not always less than $E_{r}$ and $E_{v}$ without round-off error control.

This could be because the average time-step for ERKN101217 over $10^{6}$ years is quite large. For example, with $T O L=10^{-14}, E R K N 101217$ takes a time-step of approximately 144 days on average over $10^{6}$ years, and hence, the assumption that $h y_{n-1}^{\prime}+h^{2} \sum_{j=1}^{s} b_{j} K_{j}$ is small relative to $y_{n-1}$ is invalid. Therefore, for ERKN101217, using $T O L=10^{-2 i}$, with $i=5,6,7,8$, it is not recommended to use the round-off error control technique.

\subsection{ODEX2 Integrator}

For the direct numerical solution of systems of secondorder ordinary differential equations, Hairer, Nørsett and Wanner [14] developed an extrapolation code $O D E X 2$ based upon the explicit midpoint rule with order selection and step-size control. The $O D E X 2$ integrator is good for all tolerances, especially for high precision, like $10^{-20}$ or $10^{-30}$. To observe the change in results for $E_{r}$, we performed experiments with a variety of default settings of $O D E X 2$, for example, by setting the parameter ITOL used for controlling the local error to 0 or 1 . We observed that there is hardly any significant difference in results when applied to the Jovian problem over one million years for $T O L=10^{-16}$ to $10^{-8}$.

\subsection{Step-Size Variation}

Here, we investigate the step-size variation for the variable-step-size integrators ERKN689, ERKN101217, and $O D E X 2$ applied to the Jovian problem. The eccentricities of the orbits of the Jovian planets are no more than 0.1 and there are no close-encounters between the planets. Therefore, the variable-step-size integrators should re- quire small step-size variation. Table 2 shows the stepsize variation for the above integrators applied to the Jovian problem over one million years for the local error tolerances in the range $10^{-16}$ to $10^{-8}$. The columns $h_{m n}$ and $h_{m x}$ list the percentage variation in the minimum and maximum step-sizes relative to the mean step-size. For example, $h_{m n}$ is calculated as

$$
h_{m n}=100\left(\frac{h_{\min }-\bar{h}}{\bar{h}}\right) \text {, }
$$

where, $h_{\min }$ is the smallest step-size used and $\bar{h}$ the mean step-size. For these results, we considered the onscale step-sizes by ignoring the first few step-sizes in a transient region near $t=0$ as well as the final step-size.

The step-size variation depends both upon the integrator and the tolerance chosen and ranges from approximately $-34 \%$ to $152 \%$. The largest variation between the maximum and minimum step-sizes occurs for ERKN689 with $T O L=10^{-16}$, where it is a factor of three, with $h$ ranging from $0.89 \bar{h}$ to $2.52 \bar{h}$. For the purpose of our work, we regard this variation as small. This small stepsize variation enables us to add a fixed-step-size integrator $\bar{S}-13$ (Störmer of order 13) in this paper. Therefore, we conclude that $T O L$ has little effect on the step-size variation for ERKN101217 and ODEX2.

To see the effect of round-off error, we also performed integrations with $T O L=10^{-14}$ in quadruple-precision arithmetic. The percentage variations of $h_{m n}$ and $h_{m x}$ were approximately $-18 \%$ and $133 \%$ for ERKN 689 , $-20 \%$ and $21 \%$ for $E R K N 101217$, and $-30 \%$ and $21 \%$ for ODEX2. Except for $h_{m x}$ in ERKN101217, the stepsize variations obtained in quadruple-precision arithmetic have reasonably good agreement with Table 2 . Hence the round-off error is not significant with $T O L=10^{-14}$.

\subsection{Störmer Methods}

Störmer methods are an important class of methods for solving systems of second-order differential equations. Introduced by Störmer [7], the methods have long been utilised for accurate long-term simulations of the Solar System [2]. Grazier [15] recommended an order-13, fixedstep-size Störmer method that uses backward differences

Table 2. Step-size variation for the variable-step-size integrators ERKN689, ERKN101217, and ODEX2 applied to the Jovian problem over one million years, with the local error tolerance TOL as specified in the first column.

\begin{tabular}{ccccccc}
\hline & \multicolumn{2}{c}{ ERKN689 } & \multicolumn{2}{c}{ ERKN101217 } & \multicolumn{2}{c}{ ODEX2 } \\
\hline TOL & $h_{m n}$ & $h_{m x}$ & $h_{m n}$ & $h_{m x}$ & $h_{m n}$ & $h_{m x}$ \\
\hline $10^{-8}$ & $-17 \%$ & $84 \%$ & $-20 \%$ & $23 \%$ & $-30 \%$ & $14 \%$ \\
$10^{-10}$ & $-17 \%$ & $99 \%$ & $-20 \%$ & $22 \%$ & $-13 \%$ & $12 \%$ \\
$10^{-12}$ & $-18 \%$ & $115 \%$ & $-19 \%$ & $21 \%$ & $-30 \%$ & $31 \%$ \\
$10^{-14}$ & $-18 \%$ & $134 \%$ & $-20 \%$ & $32 \%$ & $-29 \%$ & $21 \%$ \\
$10^{-16}$ & $-18 \%$ & $152 \%$ & $-34 \%$ & $71 \%$ & $-21 \%$ & $26 \%$ \\
\hline
\end{tabular}


in summed form, summing from the highest to lowest differences. The test results in [9] for simulations of the Sun, Jupiter, Saturn, Uranus and Neptune in double precision showed that the error in the energy and phase error grows as $t^{1 / 2}$ and $t^{3 / 2}$, respectively, to within numerical uncertainty when the step-size is $\left(\frac{1}{1024}\right)$-th (4.1 days) of Jupiter's orbital period. This choice of step-size ensures that the local truncation error of the Störmer method is well below machine precision. In this paper we consider the fixed-step-size Störmer method of order 13 and refer to it as the $\bar{S}-13$ integrator.

\section{Numerical Experiments for Long-Term Simulation}

First we consider the error growth in the position and velocity using the variable-step-size integrators $O D E X 2$, ERKN689, and ERKN101217. We obtained the reference solution in quadruple-precision using ERKN101217 with $T O L=10^{-18}$. To justify this particular choice for the reference solution, we integrated the Jovian problem using the ERKN101217 integrator with $T O L=10^{-20}$. The maximum difference between the positions and velocities of these two solutions is no more than $4.61 \times 10^{-13}$. We also integrated the Jovian problem in quadruple-precision with the tolerance $T O L=10^{-18}$, but using the ERKN689 integrator and found that the maximum difference with the solution for ERKN101217 with $T O L=10^{-18}$ is no more than $5.11 \times 10^{-13}$. This suggests that the ERKN101217 integrator with $T O L=10^{-18}$ is sufficiently accurate to obtain the reference solution.

Figure 1 illustrates the unweighted $L_{2}$-norm of the estimation of the maximum global error in the position as

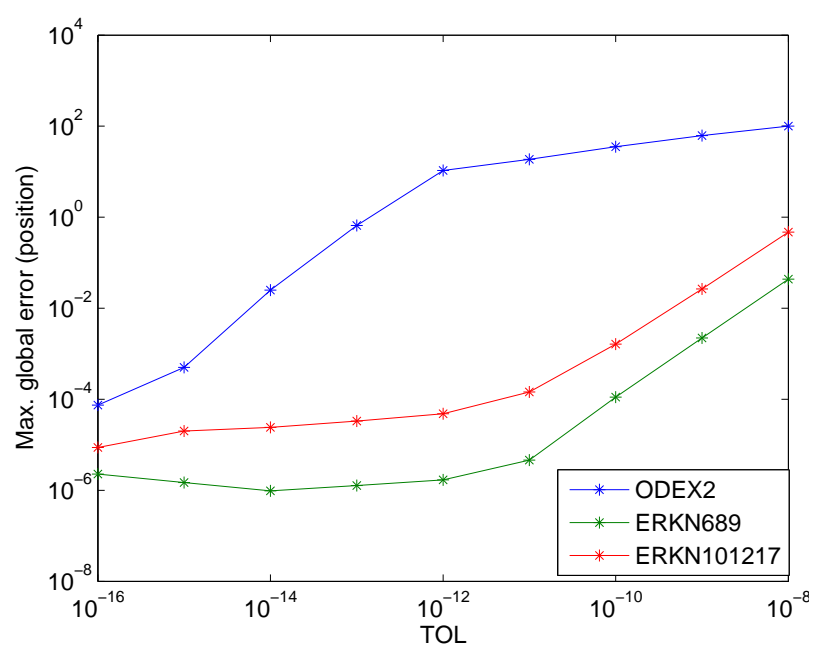

Figure 1. The maximum global error in position for the variable-step-size integrators $O D E X 2, E R K N 689$, and $E R K N 101217$ applied to the Jovian problem over one million years for local error tolerances ranging from $10^{-16}$ to $10^{-8}$. a function of tolerance with three variable-step-size integrators $O D E X 2, E R K N 689$, and ERKN101217 for the Jovian problem over one million years. In most cases, the maximum global error occurs at the end point of the integration. We chose the range $10^{-16}$ to $10^{-8}$ for the local error tolerance, because $10^{-16}$ is close to machine precision in double-precision arithmetic and tolerances greater than $10^{-8}$ lead to errors that are too large to be meaningful. The result for $O D E X 2$ is an accuracy (maximum global error) that ranges from $7.4 \times 10^{-5}$ to $1.1 \times$ $10^{2}$. We observe that the maximum accuracy (minimum of the maximum global error) is obtained with $T O L=$ $10^{-16}$ and the minimum accuracy with $T O L=10^{-8}$. The graph for $O D E X 2$ exhibits three phases: In the middle phase, with $T O L \in\left[10^{-15}, 10^{-12}\right]$ the round-off error does not yet affect the global error. Round-off has an effect for $T O L \leq 10^{-16}$, which we further investigated by using smaller tolerances, i.e, $T O L=10^{-16+k}$, for $k=0.2,0.4$, $0.6,0.8$, and 1 . As TOL decreases further from $10^{-16}$, the global error starts to increase again, which indicates the influence of the round-off error. The phase for TOL > $10^{-11}$, shows a global error of approximately $10^{1} \mathrm{AU}$, which is the diameter of Jupiter's orbit. Here, the integrator still finds the orbit but at an arbitrary position angle that could deviate as much as $180^{\circ}$. We evaluated the phase error using the formula described in Section 2 and found that it is approximately $172^{\circ}$. This means that the amplitude of the orbit is not changing, but the error in its phase angle may be as large as $\pi$.

Let us now consider the integrator ERKN101217 in Figure 1. Here, the accuracy ranges from $8.7 \times 10^{-6}$ to $4.6 \times 10^{-1}$. The maximum accuracy is again obtained with $T O L=10^{-16}$ and the minimum with $T O L=10^{-8}$. We observe that from $T O L=10^{-11}$ to $10^{-16}$ there is hardly any gain in accuracy. Therefore, if the best accuracy is required then $T O L=10^{-16}$ should be used, but otherwise, a small sacrifice in accuracy will save a considerable amount of CPU-time.

The integrator ERKN689 has an accuracy ranging from $9.7 \times 10^{-7}$ to $4.4 \times 10^{-2}$, with the maximum accuracy obtained at $T O L=10^{-14}$ and the minimum at $T O L=10^{-8}$. Therefore, nothing is gained by decreasing the tolerance from $10^{-14}$ to $10^{-16}$. The maximum at $T O L=10^{-14}$ is an indicator that the round-off error affects the global error when using tolerances between $10^{-14}$ and $10^{-16}$. To measure the possible effect of round-off error, we performed experiments in quadruple-precision. We obtained the maximum global error in the position as a function of tolerance for the local error tolerances $10^{-16}$ and $10^{-10}$ using ERKN689 and ERKN101217. We observed that both curves are straight and maintain a difference of about 1.5 orders of magnitude. In particular, the graph is not bending up for ERKN689 using the small tolerance of $10^{-16}$. This confirms the effect of round-off 
error in the double-precision arithmetic. We conclude from Figure 1 that, for local error tolerances ranging from $T O L=10^{-16}$ to $10^{-8}$, the integrator ERKN689 is the most accurate and $O D E X 2$ is the least accurate integrator.

Let us now compare this performance with the $\bar{S}-13$ integrator. Figure 2 shows the error growth in the position for the Jovian problem using the integrators $\bar{S}-13$, $O D E X 2$, ERKN689, and ERKN101217. The integration was performed over $10^{6}$ years and the error was sampled at every 100 years. The integration with the $\bar{S}-13$ integrator was performed in double-precision using a step-size of four days.

We performed two sets of experiments. For the first set of experiments, we maintained a given accuracy of approximately $10^{-4}$ for the maximum global error in the position over $10^{6}$ years. We set $T O L=10^{-16}, 10^{-10}$, and $10^{-11}$ for $O D E X 2, E R K N 689$, and ERKN101217, respectively; note that this variation in tolerance is necessary to achieve the prescribed accuracy, as illustrated in Figure 1. For small $t, E R K N 689$ and ERKN101217 are more accurate than the other two integrators, but there is a crossover approximately at $5 \times 10^{4}$ years. We see in Figure 2 that the three variable-step-size integrators achieve almost the same accuracy for the global error in position at the end of $10^{6}$ years of integration and the fixed-step-size integrator $\bar{S}-13$ achieves almost one or-

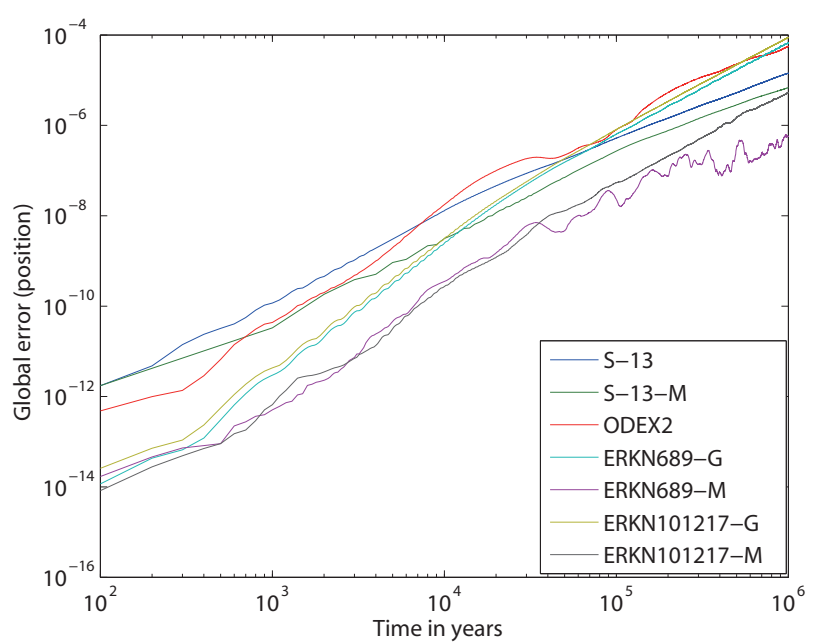

Figure 2. The error growth in position for the integrators $\bar{S}-13, O D E X 2, E R K N 689$, and ERKN101217 applied to the Jovian problem over one million years. The selection of local error tolerances is subject to a prescribed maximum accuracy. der of magnitude better accuracy than the variable-stepsize integrators.

To gain insight about the error growth depicted in Figure 2, we used unweighted linear least squares to fit a power law $\alpha t^{\beta}$ to $E_{r}$. We found that the integration error for the integrators ERKN689 and ERKN101217 grows approximately as $t^{2}$ (quadratic growth), while for $O D E X 2$ and $\bar{S}-13$ it is approximately $t^{3 / 2}$. The error growth for $O D E X 2$ is unexpected. Therefore, we repeated the integrations for $O D E X 2$ by increasing the tolerance from $T O L=10^{-16}$ to $10^{-15}$ and $10^{-14}$; then we observe approximately the quadratic error growth.

The second set of experiments for integrators $\bar{S}-13$, ERKN689, and ERKN101217, labelled S-13-M,

ERKN689-M and ERKN101217-M in Figure 2, respectively, are done such that maximum accuracy is maintained. To attain maximum accuracy, integrators ERKN689 and ERKN101217 use TOL $=10^{-14}$ and $10^{-16}$, respectively. For $\bar{S}-13$, we performed experiments with stepsize variations as shown in Table 3. We observe that $\bar{S}-13$ achieves best accuracy with a step-size of approximately 10 days. The performance of the $O D E X 2$ integrator at the prescribed accuracy, as shown in Figure 1, is also at the maximum accuracy for the local error tolerance of $10^{-16}$. When performed at the maximum accuracy, there is no longer a crossover of the $\bar{S}-13$ integrator with the integrators ERKN689 and ERKN101217. At the end of $10^{6}$ yuracyears of integration, ERKN689 achieves the best acc and ERKN101217 achieves the next best accuracy.

Some of the plots in these kinds of experiments have high-frequency oscillations. In order to smooth that data, the filter command in Matlab was employed with a window size of 50. The appropriate choice of window size is important. We have experimented (using the experiments illustrated in Figure 2 with the exclusion of those labelled S-13M, ERKN689-M, and ERKN101217-M) for values of window sizes, $0,10,20$, and 50 as shown in Figure 3. Figure 3(a) shows the result without filtering $(\mathrm{WS}=0)$. There are enough oscillations of sufficient amplitude that it is difficult to distinguish the graphs. If the window size is small, as shown in Figure 3(b) $(\mathrm{WS}=10)$ then quite a few oscillations are still there and it is not clear which of the integrators is being crossed. A window size of 50 seems to be a sensible value for this set of experiments. As is shown in Figure 3(d), it is quite clear that $\bar{S}-13$ crosses only the integrators ERKN689 and ERKN101217. We also observed (although not shown)

Table 3. The maximum global error as a function of the step-size for the fixed-step-size integrator $\bar{S}-13$, applied to the Jovian problem over one million years.

\begin{tabular}{ccccccc}
\hline Days & 4 & 10 & 15 & 20 & 25 & 30 \\
\hline Global error in position & $1.96 \times 10^{-5}$ & $1.08 \times 10^{-5}$ & $1.89 \times 10^{-5}$ & $3.95 \times 10^{-5}$ & $6.23 \times 10^{-5}$ & $1.05 \times 10^{-4}$ \\
\hline
\end{tabular}




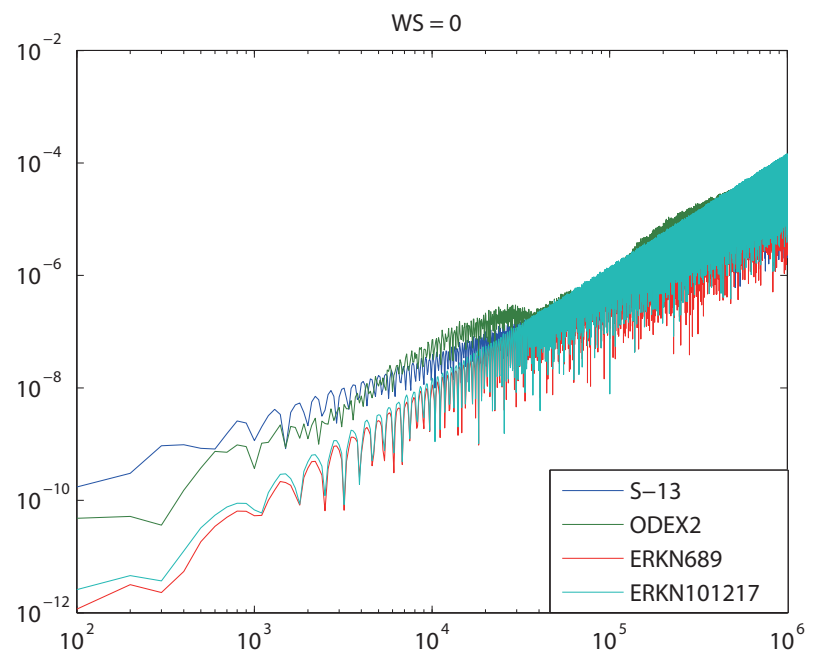

(a)

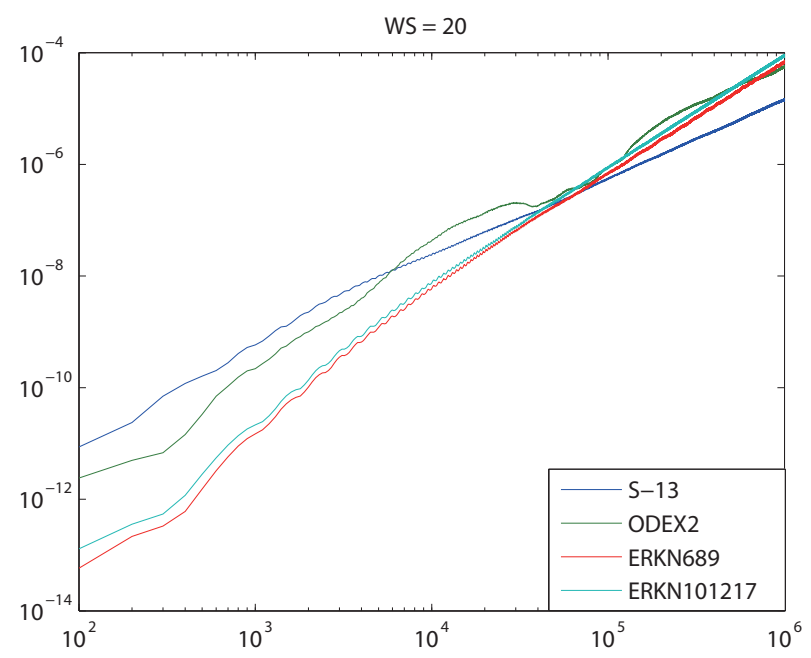

(c)

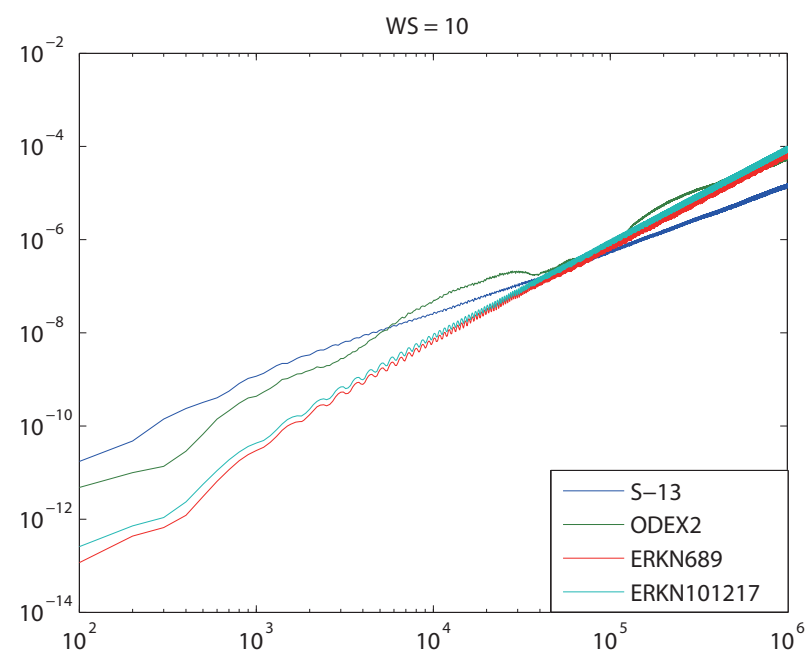

(b)

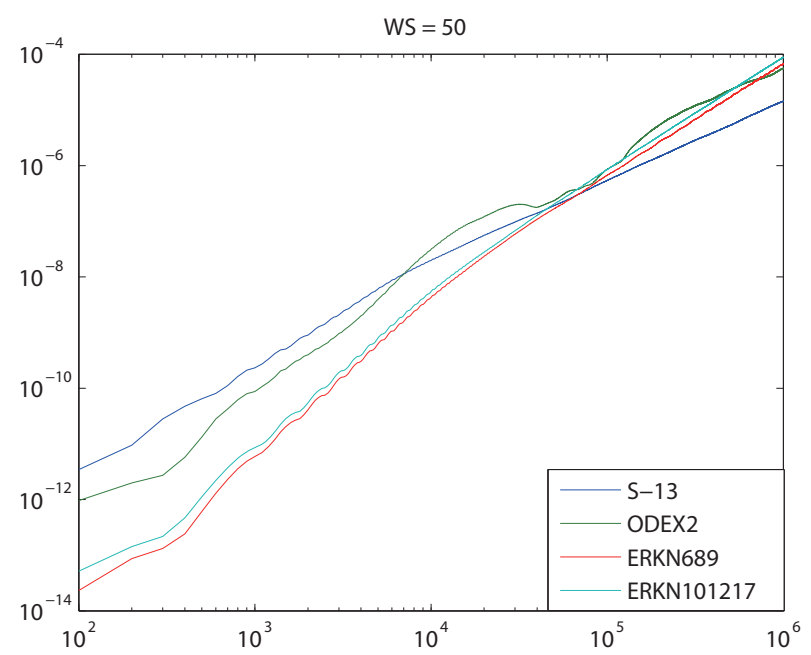

(d)

Figure 3. Experiments with a variation of the window size for the Matlab function filter. The window size is set to 0 , 10,20 and 50 in plots (a)-(d), respectively.

that filtering can complicate the interpretation of results for the first WS points, but this effect can be removed by ignoring the first WS points.

Let us now consider the accuracy of the integrators in terms of the relative error in energy and angular momentum. Figure 4 shows the error growth in the energy for the Jovian problem. The integration has been performed in double-precision over $10^{6}$ years using the same local error tolerances and integrators as for the results shown in Figure 2. For this set of experiments, we used the filter command in Matlab with a larger window size of $\mathrm{WS}=100$, because the oscillations were more pronounced than the set of experiments shown in Figure 2. The interval of integration is divided into 10,000 evenly spaced sub-intervals. To see the effect on the performance of the integrator by forcing it to hit every 100 years. We also performed experiments using ERKN101217, where we forced the integrator to hit every 50 and 200 years.
We found three parallel graphs with a maximum difference in errors at $10^{6}$ years of no more than $3.5 \times 10^{-13}$. Using 10,000 sub-intervals, we calculate the $L_{2}$-norm of the relative error in energy and angular momentum on the last accepted time step at the end of each sub-interval.

Similar to the set of experiments illustrated in Figure 2 that attain a given accuracy of $10^{-4}$, for the integrators ERKN689 and ERKN101217 (labeled by ERKN689-G and ERKN101217-G in Figure 4, respectively) we observe an error growth proportional to $t$ in energy and angular momentum. For $O D E X 2$, the error growth for energy and angular momentum shows some oscillations. The integrations were repeated for $O D E X 2$ by increasing the tolerance from $T O L=10^{-16}$ to $10^{-15}$ and $10^{-14}$, which causes the oscillations to disappear. This indicates that round-off error is the cause of the oscillations. Approximately linear error growth in energy and angular 


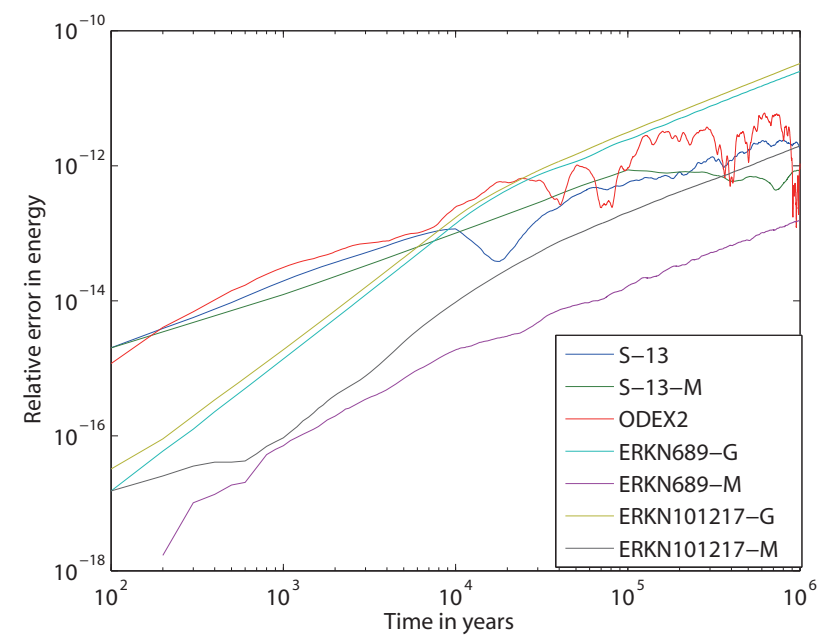

Figure 4. The error growth in the energy for the four integrators $\bar{S}-13, O D E X 2, E R K N 689$, and ERKN101217 applied to the Jovian problem over one million years. The selection of local error tolerances is subject to attaining the given and maximum accuracy.

momentum was observed particularly for $O D E X 2$ with $T O L=10^{-14}$. As in Figure 2, the integrators $O D E X 2$ and $\bar{S}-13$ with step-sizes of four days, cross the integrators ERKN689 and ERKN101217.

However, this crossover for the relative error in energy occurs at a smaller $t$ than for the global error in position. We observe from Figure 4 that, for the relative error in energy, the integrator ERKN689 using $T O L=10^{-14}$ (labeled by ERKN689-M) again achieves the best accuracy.

Let us now consider the efficiency of the integrators, which is the amount of work to attain prescribed accuracy. One way of measuring the work of different integrators is to count the number of function evaluations. Figure 5 shows plots of the number of function evaluations against the maximum global error in position, obtained for the variable-step-size integrators ERKN689, $E R K N 101217$, and $O D E X 2$, and applied to the Jovian problem over one million years with $T O L$ ranging from $10^{-16}$ to $10^{-10}$. As described in Figure 1 the best accuracy for ERKN689 is achieved at $T O L=10^{-14}$, which needs approximately 1.7 and 2.7 times more function evaluations than $E R K N 101217$ and $O D E X 2$, respectively. If we consider tolerances such that all three integrators achieve the same accuracy $10^{-4}$ then ERKN101217 is the most efficient, because it uses the least number of function evaluations. The integrator ERKN689 is approximately 2.4 and $O D E X 2$ approximately 3.3 times more expensive than ERKN101217. Our conclusion slightly changes by reducing the accuracy from $10^{-4}$ to approximately $10^{-3}$ or $10^{-2}$. The integrator ERKN101217 again achieves the best accuracy compared to the integrators $O D E X 2$ and ERKN689. For an accuracy of ap-

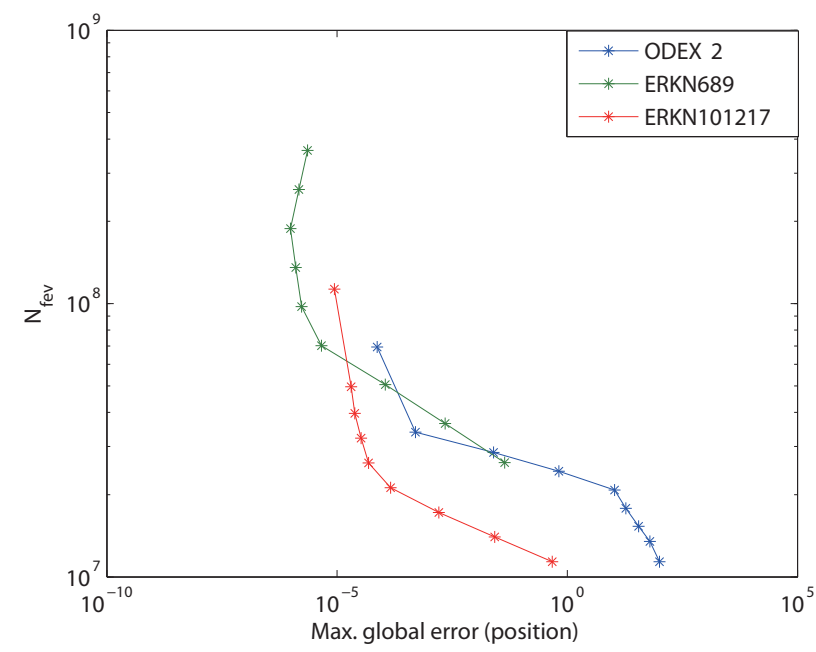

Figure 5. Efficiency plots showing the number $N_{f e v}$ of function evaluations against the $L_{2}$-norm of the maximum global error in position, obtained for the variable-step-size integrators $E R K N 689, E R K N 101217$, and $O D E X 2$, applied to the Jovian problem over one million years with $T O L$ ranging from $10^{-16}$ to $10^{-8}$.

proximately $10^{-3}$, the integrator $O D E X 2$ is approximately 1.9 and $E R K N 689$ approximately 2.1 times more expensive than ERKN101217. In contrast, for an accuracy of approximately $10^{-2}$, the integrators $O D E X 2$ and ERKN689 achieve almost the same accuracy and are approximately 2 times more expensive than ERKN101217.

We also investigated the CPU-time taken by the same variable-step-size integrators applied to the Jovian problem over one million years with local error tolerances in the range from $10^{-16}$ to $10^{-8}$. For $T O L=10^{-10}$, we found that $O D E X 2$ and $E R K N 101217$ take almost the same CPU-time, but ERKN101217 has approximately four orders of magnitude better accuracy than $O D E X 2$. For the same tolerance, ERKN689 is almost three times more expensive than ERKN101217 and $O D E X 2$, but has approximately one and five orders of magnitude better accuracy, respectively. For a given accuracy of approximately $10^{-4}, 10^{-3}$, and $10^{-2}, E R K N 101217$ takes the least CPU-time. Hence, the integrator ERKN101217 is the cheapest option.

For the given range of tolerances from $10^{-16}$ to $10^{-10}$, we found that ERKN689 achieves the best accuracy (at $T O L=10^{-14}$ ), which is approximately one and two orders of magnitude better than the best accuracies achieved by $E R K N 101217$ and $O D E X 2$, respectively. At the same point in-time, ERKN689 is almost 1.6 and 2.4 times more expensive than ERKN101217 and ODEX2, respectively. These results clearly illustrate a trade-off between accuracy and efficiency.

\section{Conclusions}

The main objective of this paper was to analyse and 
compare the efficiency and the error growth for different numerical integrators applied to the realistic problem involving the Sun and four Gas-giants. Throughout the paper, we examined the growth of the global error in the positions and velocities of the bodies, and the relative error in the energy and angular momentum of the system. The simulations were performed over as much as $10^{6}$ years.

For long-term simulations, we performed experiments to observe the error growth in the positions and velocities using the variable-step-size integrators $O D E X 2, E R K N 689$, and ERKN101217, applied to the Jovian problem over one million years for local error tolerances in the range $10^{-16}$ to $10^{-8}$. We observed that the integrators $O D E X 2$, ERKN689, and ERKN101217 attained maximum accuracy with $T O L=10^{-16}, 10^{-14}$, and $10^{-16}$, respectively. Overall, we observed that for the local error tolerances in the range $T O L=10^{-16}$ to $10^{-8}$, the integrator ERKN689 is the most accurate and $O D E X 2$ is the least accurate. We also observed that the integration error for the integrators ERKN689 and ERKN101217 grows approximately as $t^{2}$, while it grows as $t^{3 / 2}$ for $O D E X 2$ and $\bar{S}-13$. The error growth for $O D E X 2$ was unexpected. Therefore, integrations were repeated for $O D E X 2$ by increasing the tolerance from $T O L=10^{-16}$ to $10^{-15}$ and $10^{-14}$, for which we did observe the quadratic error growth.

We then investigated the efficiency of the integrators by counting the number of function evaluations against the maximum global error. We observed that the best accuracy achieved by ERKN689 uses approximately 1.7 and 2.7 times more function evaluations than $E R K N 101217$ and $O D E X 2$, respectively. Instead, if we require approximately the same accuracy of $10^{-4}$ achieved by all three integrators, the ERKN101217 is the most efficient, because it uses the least number of function evaluations. The integrator ERKN689 is approximately 2.4 and $O D E X 2$ approximately 3.3 times more expensive than

ERKN101217. We then investigated the CPU-time and observed that for a given accuracy of $10^{-4}$, the number of function evaluations is proportional to the CPU-time. Hence, also in terms of CPU-time ERKN101217 is the cheapest option, which is approximately 2.4 and 3.3 times more efficient than ERKN689 and ODEX2, respectively. For the given range of tolerances from $10^{-16}$ to $10^{-8}$, the integrator ERKN689 achieved best accuracy, which is approximately one and two orders of magnitude better than the best accuracy achieved by ERKN101217 and $O D E X 2$, respectively. At the same point in time, ERKN689 is almost 1.6 and 2.4 times more expensive than $E R K N 101217$ and $O D E X 2$, respectively. These results clearly illustrate a trade-off between the accuracy and the efficiency.

We also measured the accuracy of the integrators by obtaining the relative error in energy and angular mo- mentum. For the integrators ERKN689 and ERKN101217, the error growth is proportional to $t$, and for $O D E X 2$ with $T O L=10^{-14}$, we observe approximately linear error growth in energy and angular momentum.

\section{Acknowledgements}

The author is grateful to the Higher Education Commission (HEC) of Pakistan for providing the funding to carry out this research. Special thanks go to Dr. P. W. Sharp and Prof. H. M. Osinga for their valuable suggestions, discussions, and guidance throughout this research.

\section{REFERENCES}

[1] P. W. Sharp, "N-Body Simulations: The Performance of Some Integrators," ACM Transactions on Mathematical Software, Vol. 32, No. 3, 2006, pp. 375-395. doi:10.1145/1163641.1163642

[2] K. R. Grazier, W. I. Newman, W. M. Kaula and J. M. Hyman, "Dynamical Evolution of Planetesimals in Outer Solar System," Icarus, Vol. 140, No. 2, 1999, pp. 341352. doi:10.1006/icar.1999.6146

[3] K. Heun, "Neue Methode zur Approximativen Integration der Differentialgleichungen einer Unabhängigen Veränderlichen," Mathematical Physics, Vol. 45, 1900, pp. 2338.

[4] M. W. Kutta, "Beitrag zur Näherungsweisen Integration totaler Differentialgleichungen," Mathematical Physics, Vol. 46, 1901, pp. 435-453.

[5] F. T. Krogh, "A Variable Step Variable Order Multistep Methods for Ordinary Differential Equations," Information Processing Letters, Vol. 68, 1969, pp. 194-199.

[6] E. J. Nyström, "Über die Numerische Integration von Differentialgleichungen," Acta Societas Scientiarum Fennicae, Vol. 50, No. 13, 1925, pp. 1-54.

[7] C. Störmer, "Sur les Trajectoires des Corpuscles Électrisés," Acta Societas Scientiarum Fennicae, Vol. 24, 1907, pp. 221-247.

[8] D. Brouwer, "On the Accumulation of Errors in Numerical Integration," Astronomical Journal, Vol. 46, No. 1072, 1937, pp. 149-153. doi:10.1086/105423

[9] K. R. Grazier, W. I. Newman, J. M. Hyman and P. W. Sharp, "Long Simulations of the Outer Solar System: Brouwer's Law and Chaos," In: R. May and A. J. Roberts, Eds., Proceedings of 12th Computational Techniques and Applications Conference CTAC-2004, ANZIAM Journal, Vol. 46, 2005, pp. C1086-C1103.

[10] E. Hairer, R. I. McLachlan and A. Razakarivony, "Achieving Brouwer's Law with Implicit Runge-Kutta Methods," BIT Numerical Mathematics, Vol. 48, No. 2, 2008, pp. 231-243. doi:10.1007/s10543-008-0170-3

[11] W. H. Enright, D. J. Higham, B. Owren and P. W. Sharp, "A Survey of the Explicit Runge-Kutta Method," Technical Report, 291/94, Department of Computer Science, University of Toronto, Toronto, 1994.

[12] J. Dormand, M. E. A. El-Mikkawy and P. Prince, "Higher 
Order Embedded Runge-Kutta-Nyström Formulae," IMA Journal of Numerical Analysis, Vol. 7, No. 4, 1987, pp. 423-430. doi:10.1093/imanum/7.4.423

[13] L. F. Shampine and M. K. Gordon, "Computer Solution of Ordinary Differential Equations," W. H. Freeman, San Francisco, 1975.
[14] E. Hairer, S. P. Nørsett and G. Wanner, "Solving Ordinary Differential Equations I: Nonstiff Problems," Springer-Verlag, Berlin, 1987.

[15] K. R. Grazier, "The Stability of Planetesimal Niches in the Outer Solar System: A Numerical Investigation," PhD Thesis, University of California, 1997. 